\title{
Socio-demographic and psychosocial predictors of rheumatoid arthritis health outcome
}

Pamela Naidoo, MA (Clin Psych), DPhil

Department of Psychology, University of the Western Cape

Graham C Lindegger, MA (Clin Psych), PhD

School of Psychology, University of KwaZulu-Natal, Pietermaritzburg

Girish M Mody, MB ChB, MRCP, FCP, MD, FRCP

Department of Rheumatology, Nelson R Mandela School of Health Sciences, University of KwaZulu-Natal, Durban

Objectives. To investigate the psychosocial aspects of rheumatoid arthritis (RA) and to determine the predictors of RA health outcome in a low socio-economic group of clinic-based adult RA patients.

Design. This was a cross-sectional study. Clinic-based adult RA patients were subjected to a series of selfadministered questionnaires to assess their experience of the disease. Coping, social support, causal attribution, cognitive illness representation, pain and functional status were assessed. Joint status, which indicated the degree of severity of joint inflammation for each RA patient, was assessed by a rheumatologist.

Subjects. The sample consisted of 186 RA patients with a mean age of 49.51 years and a mean duration of RA of 10.80 years.

Outcome measures. Health status measures defined by pain and functional status, and joint status.

Results. Psychological factors, especially coping, were more significant predictors of self-report of pain and functional status than socio-demographic factors. Both socio-demographic factors and psychological factors (especially coping) were found to be significant predictors of swollen and tender joint status.
Conclusion and recommendations. As a psychological factor, coping emerged as a consistent predictor of both self-report of pain and functional status, and swollen and tender joint status. It is recommended that to enhance the quality of life of RA patients and improve their health status, the impact of psychosocial factors such as the way in which patients cope with their disease status, must be considered. Further, it is recommended that health professionals collaborate not only in attempting to refine the theoretical conceptualisation of RA, but also in devising holistic and multidisciplinary care for individuals afflicted with the disease.

Rheumatoid arthritis (RA) is a systemic autoimmune disorder of unknown aetiology, which is disabling and chronic. The synovial membranes of multiple joints are usually affected, with the formation of chronic synovitis leading to bone and cartilage damage.' The most common outcome of the established disease is the progressive development, in varying degrees, of joint destruction, deformity and disability. The primary cause of RA is unknown and it has, therefore, been described as 'one of modern medicine's major enigmas'.

There has been a proliferation of studies from developed countries on the medical and psychological aspects of RA, and more recently on the psychoneuroimmunological aspects of the disease. RA is a chronic and disabling disease with serious clinical, psychosocial and economic effects. Despite investigation into ways of improving the quality of life of individuals with RA, it is difficult to contain the overall cost of this debilitating disease. One possible explanation for not being able to control the economic effects of the disease rests on the unclear aetiology of RA. Attempts to prevent the disease from manifesting have been futile thus far. Consequently, the cost of the disease to the individual, his or her workplace and to the health care system remains high.

Most RA research in South Africa is medically based ${ }^{3,4}$ com- 
pared with international research trends in the area of chronic illness. International research has become increasingly focused on the importance of both socio-demographic and psychosocial factors in disease outcome. ${ }^{5}$ This South African study appears to be the first to conduct a comprehensive investigation into the way in which psychosocial factors are associated with socio-demographic factors, disease factors, and health-related quality of life. A sample obtained from a developing country such as South Africa, with its unique sociological patterns around family structure, cultural practices, religious beliefs and economic disparity between different groups of people, differs from a sample based in a developed country such as North America.

Psychosocial and biomedical disease factors often account for variance in disease outcome. Although results from studies have varied, general support has been found for the diseasecourse hypothesis that takes into account the psychological response to RA experience. For example, there is often a poor correlation between disease status and disability in RA patients. ${ }^{\circ}$ Frequently, patients with severe RA as measured by clinical assessments including $X$-rays and other radiographic measures, experience mild disability, whereas patients with mild disease present with severe disability. ${ }^{7}$ It is important, therefore, to identify other variables responsible for affecting the variability in the health outcome measures of these patients. Psychological factors such as coping, social support, causal attribution, and cognitive illness representation are known to impact on health outcome. ${ }^{8}$

The aim of this study was to investigate the predictive value of both socio-demographic and psychosocial factors in RA health outcome.

\section{Methods}

This was a cross-sectional study. A purposive or judgemental non-probability sampling method ${ }^{9}$ was used to obtain a sample of RA patients. One hundred and eighty-six adult female and male RA patients were selected over an 18-month period from the outpatient rheumatology clinic at King Edward VIII Hospital, Durban.

Permission to conduct the study was obtained from the relevant authorities at the University of KwaZulu-Natal's Nelson R Mandela School of Health Sciences and King Edward VIII Hospital. Approval from the Ethics Committee at the medical school was also obtained.
Only two rheumatologists from the Department of Rheumatology were responsible for medically examining the subjects and confirming a diagnosis of RA, which ensured that there was consistency in applying the relevant medical criteria in diagnosing RA, and in assessing the extent to which each subject was functionally impaired (disabled as a result of RA).

The clinical records were reviewed before the patients were enrolled in the study. The rheumatologists at the clinic assessed patients who had been previously diagnosed with RA, and who were able to read in English. The rheumatologist reviewed the clinical records of the patients, once again, to determine whether these patients fulfilled the American Rheumatism Association (ARA) ${ }^{10}$ criteria for the diagnosis of RA. To ensure a valid and reliable sample only those patients who fulfilled the criteria were approached to participate in the study. Patients with a co-existing serious medical or psychiatric condition were excluded. The purpose of the study and the requirements were discussed in detail with the patients and once their informed consent was obtained they were enrolled as participants. Participants were also informed of their right to withdraw from the study.

Information pertaining to the subjects' socio-demographic status, criteria in accordance with the American College of Rheumatology ${ }^{11}$ disease activity measures, assessment of function, and psychological measures were obtained and recorded. The classification of the patients' functional status according to the ARA revised criteria was also recorded (class I - class IV). ${ }^{11}$

Senior nursing sisters were responsible for the collection of blood samples from each patient. These were then forwarded to the hospital laboratory for analysis. Once the results were obtained they were interpreted and noted by the two rheumatologists involved in the study.

A series of questionnaires were administered to the subjects. A semi-structured interview also formed part of the assessment procedure. A nursing sister at the rheumatology clinic acted as an interpreter for black patients who felt they could express an idea or thought more clearly in an indigenous language such as Zulu or Xhosa. A detailed biographical inventory was administered and the actual number of years of RA was recorded. Psychosocial measures and health outcome measures were also obtained. 
The psychosocial measures included the London Coping with Rheumatoid Arthritis Questionnaire (LCRAQ), a 36-item questionnaire based on a Likert-type format, ${ }^{12}$ the IIness Specific Social Support Scale (IS-SSS) used to assess the construct of problematic support and positive social support in individuals with RA, $^{13}$ and causal attribution and cognitive illness representation. A semi-structured questionnaire was devised to assess the causes that RA patients attribute to their disease and to assess RA patients' cognitive illness representations. 'Open-ended' responses were obtained to ascertain the causal attribution subjects made at the time of disease onset and at the time the study was conducted.

Measures of health outcome included measures of pain and functional status. Joint status was retained as a separate measure. Pain was measured using the Visual Analogue Scale (VAS) $^{14}$ and the Arthritis Impact Measurement Scale 2 (AIMS2), ${ }^{15}$ a pain subscale. Disability was measured using the VAS, AIMS2, and the Stanford Health Assessment Questionnaire (HAQ). ${ }^{16}$ Joint status was measured using the 28-joint count. ${ }^{17}$

The individual measures of pain and functional assessment measures (namely measures of disability and joint count) in this study were factor analysed, and two measures emerged. The first was the measure of pain and disability, and the second was the 28-joint count measure. Pain and disability, a dependent variable (DV) in this study, was therefore retained as a single measure in the statistical analyses.

\section{Results}

The age range of this hospital-based outpatient clinic sample was 20 - 80 years, with a mean age of 49.51 years and a standard deviation (SD) of 10.86 years. The mean number of years of RA was 10.80 (SD 7.96). As shown in Table I, the majority of participants were women (87.1\%), a little more than half the sample (54.8\%) had never been married, the majority (81.2\%) had at least a senior primary or high school education, most participants were not engaged in paid work activities, and well over half the subjects reported a monthly household income of less than R1 000. Most participants identified themselves as being either Hindu or Christian. Indian South Africans comprised $65.6 \%$ of the sample.

In order to test the hypotheses, the data were statistically

\begin{tabular}{|c|c|c|}
\hline $\begin{array}{l}\text { Demographic variables } \\
\text { Age (yrs) }\end{array}$ & Frequency & Percentage (\%) \\
\hline $20-29$ & 11 & 5.9 \\
\hline $30-39$ & 21 & 11.3 \\
\hline $40-49$ & 54 & 29.0 \\
\hline $50-59$ & 69 & 37.1 \\
\hline$\geq 60$ & 31 & 16.7 \\
\hline \multicolumn{3}{|l|}{ Marital status } \\
\hline Married & 45 & 24.2 \\
\hline Never married & 102 & 54.8 \\
\hline Widowed & 26 & 14.0 \\
\hline Separated or divorced & 13 & 7.0 \\
\hline \multicolumn{3}{|l|}{ Sex } \\
\hline Male & 24 & 12.9 \\
\hline Female & 162 & 87.1 \\
\hline \multicolumn{3}{|l|}{ Educational level } \\
\hline No formal schooling & 22 & 11.8 \\
\hline $\begin{array}{l}1-3 \text { years of junior school } \\
4-6 \text { years senior }\end{array}$ & 4 & 2.2 \\
\hline primary school & 26 & 14.0 \\
\hline $1-3$ years of high school & 71 & 38.2 \\
\hline 4 - 6 years of high school & 54 & 29.0 \\
\hline Tertiary education & 9 & 4.8 \\
\hline \multicolumn{3}{|l|}{ Employment status } \\
\hline Employed & 44 & 23.7 \\
\hline $\begin{array}{l}\text { Not engaged in paid } \\
\text { work activities }\end{array}$ & 142 & 76.3 \\
\hline \multicolumn{3}{|l|}{ Monthly household income } \\
\hline$<$ R1000 & 109 & 58.6 \\
\hline R1 $000-$ R2 000 & 58 & 31.2 \\
\hline R2 $000-R 6000$ & 16 & 8.6 \\
\hline$>\mathrm{R} 6000$ & 3 & 1.6 \\
\hline \multicolumn{3}{|l|}{ Religion } \\
\hline Christian & 86 & 46.2 \\
\hline Hindu & 82 & 44.1 \\
\hline Muslim & 17 & 9.1 \\
\hline \multicolumn{3}{|l|}{ Racial classification } \\
\hline Black & 64 & 34.4 \\
\hline Indian & 122 & 65.6 \\
\hline Total & 186 & 100 \\
\hline
\end{tabular}

analysed using the SPSS statistical programme. ${ }^{18}$ Correlational analysis was used to test the linear relationships between the socio-demographic variables, psychosocial variables and health outcome of RA patients. Hiearchical multiple regression analysis was used to test the health-sustaining function of psychosocial factors (referred to as the directeffects hypothesis). ${ }^{19}$ 
There were significant positive correlations between internal control ( $r=0.22)$, external control $(r=0.16)$ and self-report of pain and functional status. This result indicates that subjects who believed that they had the ability to control the disease as well as those who believed that RA can only be controlled by factors outside the individual had increased levels of pain and poorer functional status.

Significant negative correlations were found between total coping and self-report of pain and functional status $(r=-0,30)$, proactive coping and self-report of pain and functional status $(r=-0.21)$, negative-internal coping and self-report of pain and functional status $(r=-0.39)$; and positive-interactive coping and self-report of pain and functional status ( $r=-0.20)$. These associations indicate that increased ability to cope with RA was related to lower scores on pain and functional status. In addition, proactive and positive-interactive coping styles of the subjects were related to decreased levels of pain and better functional status. Finally, a somewhat contradictory finding indicates that a negative-internal coping style was associated with lower pain scores and better functional status.

As shown in Table III, significant positive relationships were found between internal control and swollen and tender joint status $\mid r=$ $0.16)$ and external control and swollen and tender joint status ( $r$ $=0.15$ ). Although these findings indicate small but significant correlations, it demonstrates that despite the subjects' belief that they are able to control the disease they still have increased joint involvement. In addition, those subjects who believed factors out side the individual played a greater role in controlling the disease also had increased joint involvement. Psychological control, therefore, may not be as significant as biological factors during symptom flare-ups, or it is possible that psychological factors played a role in an unexpected direction. Whether there is a perception of greater internal or external control in this sample did not make a difference to joint involvement.

The only significant correlation with coping variables was found between negative-internal coping and joint status ( $r=-0.27)$ indicating that an increased negative style of coping was associated with poor joint status.

\section{Multivariate analysis}

Multivariate analysis was conducted to construct predictive models of the factors that contribute to each of the DVs associated with RA, namely self-report of pain and functional status, and swollen and tender joint status. A hierarchical method of analysis was employed whereby the variance associated with factors which deemed causally prior, which have been termed IVs, was partialled out before the effect of other psychological variables was examined. A mixture of analysis of variance and hierarchical multiple regression analysis ${ }^{21}$ was used to isolate the predictors of each of the two DVs, namely pain and functional status. A two-step procedure was used to develop a model for each dependent variable.

\section{Step one}

All the IVs found to be significantly related to the DV in the bivariate analysis were entered into the regression analysis, and a stepwise procedure was employed to determine which variable remained in the final model

\section{Step two}

All the psychological variables found to be significantly related to the DV in the bivariate analysis were entered into the regression analysis next, as a set, after the IV. A stepwise procedure was employed once again to determine which variable remained in the final model.

By means of this two-stage process it was possible to develop predictive models for each DV, where the psychological variables were only entered once the variance for the socio- demographic variables had already been partialled out.

There was one minor difficulty in developing the models. There were two sets of categorical variables among both the IVs (marital status and religion) and the psychological variables ICA_O and CA_R — causal attributions made by the subjects at the time of onset of the disease and causal attributions made at the time the study was conducted, respectively) which could not simply be entered into the regression analysis. On completion of step one, regression residuals were computed, and these were used as DVs in two one-way ANOVAS, to determine whether marital status and religion were also significant predictors. In no instances were these found to be significant.

The results of the first analysis are reported in Table IV.

The results of the multiple regression analyses for each of the DVs are presented below.

\section{Regression models}

\section{Predictive model one: self-report of pain and func- tional status}

The partial correlations (partial r) presented in Table IV indicate which socio-demographic and psychological variables are sig- 
Table IV. Regression equations for self-report of pain and functional status, and swollen and tender joint status

\begin{tabular}{|c|c|c|c|}
\hline Significant predictors & $\beta$ & $\dagger$ & Partial R \\
\hline Income & $-0.16^{*}$ & -2.49 & -0.18 \\
\hline Employment status & $0.14^{*}$ & 2.20 & 0.16 \\
\hline Negative-internal coping & $-0.33 \dagger$ & -5.20 & -0.36 \\
\hline Total coping & $-0.26 \dagger$ & -4.20 & -0.30 \\
\hline \multicolumn{4}{|c|}{ DV: Swollen and tender joint status $\left(R^{2}=0.90 F(2.183)=9.05\right)$} \\
\hline Significant predictors & $\beta$ & $\dagger$ & Partial R \\
\hline Employment status & $0.13^{*}$ & 1.86 & 0.14 \\
\hline Negative-internal coping & $-0.25 \dagger$ & -3.47 & -0.25 \\
\hline
\end{tabular}

nificant predictors of the DV self-report of pain and functional status.

The demographic variables found to be significant predictors of the DV self-report of pain and functional status were employment status and monthly household income.

The results of the one-way ANOVAS indicated that there was a significant difference between the never-married, married, widowed and divorced groups on self-report of pain and functional status (F $=2.86, p<0.05$ ). Post hoc analysis using the Schéffe test revealed that there is a significant difference between the married and widowed groups on selfreport of pain and functional status, with the married group reporting lower levels of pain and better functional status $15 \%$ level of significance) than the widowed group.

A significant difference was also found between the different religious groups, i.e. Christians, Hindus and Muslims on selfreport of pain and functional status ( $F=3.39, p<0.05$ ). Use of the Schéffe test revealed that Muslims and Christians were significantly different on self-report of pain and functional status (5\% level of significance). Muslims reported lower levels of pain and better functional status.

The two psychological variables, negative-internal coping and total coping were found to be significant predictors of self-report of pain and functional status.

The psychological variables that could not be entered into the regression equation were causal attribution at the time of onset (CA_O) of the illness and causal attribution at the time the study was conducted (CA_R). The results of the ANOVAS with CA_O and CA_R on self-report of pain and functional status were not significant

Monthly household income, employment status, racial classification, negative-internal coping and total coping were entered into a stepwise multiple regression. The residuals (residual one: income, employment status and racial classification) were then saved as DVs. ANOVAs were then computed between residual one (treated as a DV) and marital status and religion. As already stated, marital status and religion had significant F-values with the DV self-report of pain and functional status. A significant difference was found between the married and widowed group $(F=3.26, p<0.05)$ and residual one, which was in turn saved as residual two. The effect for religion was not significant.

Once residual two was ascertained the psychological variables total coping, proactive coping, negative-internal coping, positive-interactive coping, internal control and external control were entered into a stepwise regression on residual two as the DV. In the final analysis employment status, monthly household income, negative-interactive coping and total coping were found to be significant predictors of self-report of pain and functional status. A beta coefficient of -0.16 indicates that higher monthly household income was predictive of less severe pain and better functional status. Unemployed status (not being engaged in paid work activity) was predictive of increased levels of pain and poor functional status (beta coefficient 0.14). The beta coefficient -0.42 indicates that increased negative-internal coping was predictive of lower degrees of self-report of pain and better functional status. A beta coefficient of -0.26 indicates that better overall coping was predictive of lower levels of reported pain and better functional status. 


\section{Predictive model two - swollen and tender joint status}

The only demographic variable found to be a significant predictor of swollen and tender joint status was employment status. ANOVA computed for marital status, racial classification, and joint status did not yield significant results. ANOVAs computed for CA_O and CA_R, and swollen and tender joint status, were also not significant.

Employment and negative-internal coping were entered into a stepwise multiple regression analysis to assess the variables' power to predict swollen and tender joint status. Both variables were found to be significant predictors of swollen and tender joint status, although negative-internal coping was found to be a more significant predictor of swollen and tender joint status (beta coefficient -0.25). Increased negative-internal coping style, therefore, was predictive of lower levels of swollen and tender joint status. Being unemployed or not being engaged in paid work activities was predictive of poor joint status (beta coefficient 0.13 ).

\section{Discussion}

\section{Relationship between socio-demographic factors and RA health outcome}

In this investigation, those RA patients who were not engaged in paid work activities reported higher levels of pain and poorer functional status. These patients also reported poor joint status as measured using the 28 -joint count. A possible reason for these findings is that those patients who were not employed spent more time dwelling on their pain, limited functional ability and degree of joint inflammation. The fact that these RA patients' had limited economic resources may have been another reason why they were at risk for poor health status.

It is widely accepted that individuals with few resources are at risk for illness onset. ${ }^{22}$ In RA, socio-economic decline is associated with a greater prevalence of the disease. ${ }^{23}$ The ERAS study group based in the UK, which investigated socioeconomic deprivation and RA, found that socio-economic factors were associated with a worse clinical course of rheumatoid disease. ${ }^{24}$ Individuals in the study who came from deprived circumstances were found to have more severe disease, as assessed by the HAQ and joint scores. Furthermore, it was reported that women appeared to be more at risk. ${ }^{24}$
Previous research has confirmed that those individuals engaged in paid work have better health status. ${ }^{25-27}$ Being able to work despite having a disease helps to increase an individual's self-worth, thereby buffering or reducing existing symptoms such as pain and depression. Research outcomes also report an increased incidence of pain and depression among women with RA who have suffered work loss, face the threat of work loss, or who are work disabled, and suggest that these outcomes are related more to a loss of social role than to disease activity that has disrupted work. ${ }^{28}$

\section{Relationship between psychosocial factors and RA outcome}

\section{Social support and RA outcome}

The hypothesis stating that there would be a significant association between perceived social support and RA outcome was rejected. These findings have not been replicated in other studies. Social support has generally been associated with increased psychological well-being and better functional status in chronically ill individuals. ${ }^{120}$ Social support may also be perceived as problematic, with resulting negative effects. ${ }^{29}$ It is apparent, therefore, that social support does not only have general, positive effects. The reasons why the findings in this study are not corroborated by the existing literature are unclear and deserve further investigation.

\section{Internal and external control and RA outcome}

In support of the hypothesis pertaining to the relationship between the psychological and RA health outcome factors, it was found that increased perceived internal and increased perceived external control were associated with an increased level of pain and poor functional status. The finding regarding perceived external control is somewhat surprising because it would be expected that individuals with a greater degree of perceived internal control would report less severe symptoms compared with individuals with a perceived external sense of control. A similar finding was obtained for both perceived internal and perceived external control and swollen and tender joint status. A possible explanation for this is that during periods when symptom flare-ups are extremely severe and overwhelming, the resultant effect is the experience of severe pain, poor functional status, and poor joint status, regardless of the patients' locus of control (i.e. internal or external control). 


\section{Coping and RA outcome}

In this study an inverse relationship was found between overall coping, proactive coping, negative-internal coping and positive-interactive coping, and RA patients' self-report of pain severity and functional status. These results (except the relationship between negative-internal coping and pain and functional status) are consistent with what is reported in the literature about the positive relationship between active coping strategies and psychological well-being and functional status. ${ }^{30}$ Passive or avoidant coping strategies are reported to be negatively correlated with RA outcome. ${ }^{30}$ These reports suggest that individuals who directly confront their illness have better illness outcome. The finding in this study, therefore, that negative-internal coping is significantly associated with lower pain scores and better functional status is unexpected. One possible explanation for this finding lies in intrapsychic factors. Those RA patients who use a negative-internal style of coping marked by avoidance behaviour and the need for 'privacy' may have underscored lon the self-report measures of pain and functional abilityl their level of pain, and not adequately reflected the difficulty they experienced with regard to their functional ability. The positive association found between negative-internal style of coping and poor joint status (increased count of swollen and tender joints) is consistent with findings associating poor joint status with poor functional status. ${ }^{30}$

\section{Predictive models}

\section{Model one - predictors of self-report of pain and functional status}

The socio-demographic factors (employment status and monthly household income) and the psychological factors (total coping and negative-internal coping) were found to be significant predictors of self-report of pain and functional status. Higher monthly household income was predictive of low levels of pain and better functional status, and not being engaged in paid work activity was predictive of increased levels of pain and poor functional status. Good overall coping and high negative-internal coping were found to be predictive of decreased pain levels and better functional status. The factor negative-internal coping, marked by avoidance of social interaction, was one of the factors obtained by subjecting the London Coping with Rheumatoid Arthritis Questionnaire to factor analysis.

The finding that socio-demographic factors were significant predictors of RA health status was expected. Having a low socio-economic status implies that limited social, economic and other resources are available. The subjects in this study were not only of low socio-economic status but also lived with the challenge of a chronic illness. RA requires that the afflicted individual have adequate resources, including access to and the ability to afford medical care in order to manage the disease. In a review of the socio-economic context of health in South Africa, researchers emphasise the point that domains outside the health sector affect health status. ${ }^{31}$ They report that marginalised groups such as the poor are particularly affected. Poor women and children are also reported to be at risk. The health status of RA patients in this study, therefore, was compromised by their low socio-economic status. The fact that a greater number of women are diagnosed with RA also deserves special attention in light of this report. ${ }^{31}$ If the health of poor women is at risk, then the health status of the subjects in this study, the majority of whom were women and already afflicted with a chronic disease, continues to be at risk.

The finding regarding the predictive value of coping in relation to RA outcome corroborates with previous research findings that psychological factors play a significant role in mediating RA and its outcome. ${ }^{30}$ However, the finding that increased negative-internal coping is predictive of reports of decreased pain and better functional status, is not consistent with studies reported in the literature. Previous studies have shown that negative and avoidant cognitive coping strategies are associated with poorer outcome, while active strategies such as 'information-seeking' and 'active coping' have been associated with better outcome. ${ }^{32}$ In a study of male and female RA patients it was found that the best predictors of high pain levels were both physical disability and passive coping. ${ }^{33}$ It is clear, therefore, that the finding in this study with regard to the predictive power of negative-internal coping on pain and functional status is not consistent with these findings. However, as suggested earlier, it is possible that those RA patients using negative-internal coping strategies are private copers who avoid social interaction but have the necessary psychological resources to cope with their experience of pain and functional limitation. In addition, the sample in this study differed from samples in studies conducted in developed countries by virtue of the fact that the study was conducted in South Africa, a developing country, among subjects of low socio-economic status. Socio-economic status, social and cultural factors are expected to influence personality development. Personality would be one psychological factor among many that would influence the relationship between an individua's appraisal of a stressor such as RA, and the individual's choice of coping strategies to deal with the illness. The fact that negative-internal coping was found to be a significant predictor of pain intensity and functional 
status in this study requires further investigation. Specifically, the impact of socio-economic status on personality development, as well as the impact of personality on psychological coping resources and ultimately health outcome in RA and other chronic diseases, need to be addressed.

\section{Model two - predictors of swollen and tender joint status}

Hierarchical multiple regression was conducted to obtain the significant predictors of swollen and tender joint status. The socio-demographic variable employment status and the psychological variable negative internal-coping were found to be significant predictors of swollen and tender joint status. RA patients in this study who were not involved in paid work activities were more likely to have poor joint status. Increased negative-internal coping was predictive of decreased joint involvement (better swollen and tender joint status).

Numerous explanations could be offered for the finding regarding employment status and swollen and tender joint status. One possible explanation is related to the gender imbalance of the sample, as the majority of the subjects were women. The unemployed women in the sample were homemakers who were frequently involved in physically demanding household tasks, which may have put a strain on their joints, thereby worsening their joint status. The finding of the predictive value of employment status with regard to joint status, may also serve to confirm the established relationship between low socio-economic status and poor health outcome. ${ }^{34}$ It is expected that individuals with limited economic resources will also have limited social, psychological and other resources needed to enhance coping with a chronic illness such as RA, resulting in poor health status.

An unusual and unexpected finding was that increased negative-internal coping predicted better swollen and tender joint status, a finding similar to that found for pain and functional status. Decreased joint involvement was predicted for those RA patients who privatised their coping and avoided social interaction. As mentioned previously the use of avoidant coping is usually associated with poor health outcome, ${ }^{30}$ a relationship that was not confirmed in this study. The finding regarding negative-internal coping as a predictor of joint functioning parallels the finding with pain and functional status as an outcome measure. Investigating the pathways between socio-economic status, psychological factors (including personality) and health outcome among low socio-economic individuals with RA is required.

\section{Conclusion}

Clearly, a combination of biological, psychological, social and economic variables affect the wellbeing of RA patients.
Understanding the relationships and interrelationships between these variables is critical in planning a multidisciplinary intervention programme(s) for RA patients. Because of the complexity of the disease, the poorly understood nature of its aetiology, and the physical and psychological consequences experienced by individuals with RA, it is imperative that rheumatologists, nurses, psychologists and other health professionals collaborate in researching not only medical and psychological aspects of the disease, but also in planning programmes to assist individuals with this condition.

\section{Recommendations}

It is recommended that psychological factors be considered important mediators between RA and RA health outcome. Ultimately, psychological factors are crucial in contributing to a RA patient's quality of life.

More importantly, this first comprehensive South African study of RA taking psychological factors into account should be used to create a research agenda that aims to explore RA in its multidimensional context. Based on the findings of this study it is recommended that negative-internal coping requires further investigation because it was found to be a significant predictor of pain intensity and functional status. In addition, the impact of socio-economic status on personality development, as well as the impact of personality on psychological coping resources and ultimately health outcome in RA and other chronic diseases, also needs to be addressed. Finally, the unusual finding in this study that social support does not impact on health outcome is worthy of further investigation.

\section{References}

1. Shearn MA, Hellman D. Arthritis and musculoskeletal disorders. In: Schroeder SA Krupp MA, Tierney LM, McPhee SJ, eds. Current Medical Diagnosis and Treatment. Connecticut: Appleton \& Lange, 1990: 544-584

2. Buchanan WW. Rheumatoid arthritis: ano her new world disease? Semin Arthritis Rheum 1994; 23: 289- 294.

3. Mody GM, Shaw J, Ramchurren A. Rheumatoid ar hritis impact survey. S Afr Med 1988; 74: 409- 410 .

4. Mody GM, Meyers OL. Rheumatoid arthritis in Blacks in Sou h Africa. Ann Rheum Dis 1989; 48: 69-72.

5. Holm MB, Rogers JC, Kwoh CK. Predictors of functional disability in patients with heumatoid arthritis. Arthritis Care and Research 1998; 11: 346-355.

6. Anderson KO, Bradley LA, Wise CM. Rheumatoid arthritis: review of psychological factors related to etiology, effects and treatment. Psychol Bull 1985; 98: 358-387.

7. Newman S, Revenson TA. Coping with heumatoid ar hritis: Psychological aspects of heumatic disease. In: Newman S, Shipley M, eds. Baillière's Clinical Rheumatology: International Practice and Research. London: Baillière Tindall, 1993.

8. Shipley M. Newman SP. Psychological aspects of heumatic diseases. In: Newman $S$, Shipley M, eds. Baillière's Clinical Rheumatology: International Practice and Research. London: Baillière Tindall, 1993

9. Bless C, Higson-Smith C. Fundamentals of Social Research Methods. An African Perspective. 2nd ed. Cape Town: Juta \& Co., 1995

10. American Rheumatism Association. Revised Diagnostic Criteria for Rheumatoid Ar hritis, 1987

11. American College of Rheumatology. Revised Criteria for the Classification of Rheumatoid Arthritis, 1987 
12. Newman S, Fitzpatrick R, Lamb R, Shipley M. Patterns of coping in rheumatoid ar hritis. Psychology and Health 1990; 4: 187-200.

13. Revenson TA, Majerovitz SD. Illness-specific social support scale. Patient and marital adaptation to heumatic disease study. 1992.

14. Melzack R. Concepts of pain measurement. In: Melzack R, ed. Pain Measurement and Assessment. New York: Raven Press, 1983.

15. Meenan R, Mason J, Anderson J, Guccione A, Kazis L. The content and properties of a revised and expanded ar hritis impact measurement scales health status questionnaire. Arthritis Rheum 1992; 35: 1-10.

16. Fries J, Spitz P, Kraines RG, Holman HR. Measurement of patient outcome in ar hritis. Arthritis Rheum 1980; 23: 137-145.

17. Fuchs HA, Brooks RH, Callahan LF, Pincus T. A simplified twenty-eight-joint quantito tive articular index in heumatoid arthritis. Arthritis Rheum 1989; 32: $531-537$

18. Norusio MJ. SPSS: Base Systems User's Guide 1992. Chigaco: SPSS Inc, 1992

19. Shumaker SA, Brownell A. Toward a theory of social support: Closing conceptual gaps. Journal of Social Issues 1984; 40: 11-36

20. Ward MM, Leigh JP. Marital status and the progression of functional disability in patients wi h rheumatoid arthritis. Arthritis Rheum 1993; 36: 581 - 588 .

21. Cohen J, Cohen P. Applied Multiple Regression/Correlational Analyses for the Behavioural Sciences. New Jersey: Earlbaum, 1988.

22. Berkman LF, Syme SL. Social networks, host resistance, and mortality : a nine-year follow up study of Almeda County residents. Am J Epidemiol 1979; 109: 186 204.

23. Mitchell JM, Burkhauser RV, Pincus T. The importance of age, education, and co-morbidity in he substantial earnings losses of individuals wi h systemic polyar hritis.
Arthritis Rheum 1988; 31: 348

24. Young A, Wilkinson P, Talamo J, et al. Socio-economic deprivation and heumatoid disease: what lessons for the hea h service? Ann Rheum Dis 2000; 59: 794-799.

25. Nathanson C. Social roles and hea th status among women: the significance of employment. Soc Sci Med 1980; 14A: 463-471.

26. Rosenfield S. The effects of women's employment: personal control and six differ ences in mental hea h. J Health Soc Behav 1989; 30: 77-91

27. Verbrugge L. Physical heal h of clerical workers in the U.S., Farmingham and Detroit Womens Health 1984; 19: 17-41.

28. Fifield J, Reisine ST, Grady K. Work disability and the experience of pain and depression in heumatoid arthritis. Soc Sci Med 1991; 33: 579-585.

29. Dwyer KA. Psychosocial factors and heal $h$ status in women wi h rheumatoid ar hritis: predictive models. Am J Prev Med 1997, 13: 66-72.

30. Newman S, Mulligan K. The psychology of heumatic diseases. Baillieres Clin Rheumatol 2000; 14: 787-795.

31. Lund F, Patel Z. Socio-economic context of heath. In: David H, ed. South African Health Review. Durban: Health Systems Trust, 1995.

32. Manne S, Zautra A. Spouse criticism and support: heir association wi h coping and adjustment among young women with heumatoid ar hritis. J Pers Soc Psychol 1989 56: $608-617$

33. Covic T, Adamson B, Hough M. The impact of passive coping on rheumatoid arthritis pain. Rheumatology 2000; 39: 1027-1030.

34. Marks DF, Murray M, Evans B, Willig C. Health Psychology. Theory, Research and Practice. London: SAGE Publications, 2000 